\title{
Analytical and Finite Element Analysis of Thermal Autofrettage process in Spherical vessel
}

\author{
Rupali $^{1}$, S. C. Mondal ${ }^{2}$, S. Sarkar ${ }^{2}$ \\ ${ }^{1}$ (Research Scholar, Department of Mechanical Engineering, Jadavpur University, India) \\ ${ }_{2}^{2}$ (Faculty, Department, of Mechanical Engineering, Jadavpur University, India)
}

\begin{abstract}
The mechanism of thermal autofrettage is described in relation to the more common mechanical autofrettage. This paper provides analytical and FE analysis of thermal autofrettage process has been conducted for spherical vessel considering von Mises yield criterion to predict behavior within the plastic zone. Elastic-plastic stress distribution subjected to thermal load are evaluated. This study include residual stress prediction on an uniaxial loading-unloading stresses of elastic perfectly plastic material model. Feasibility of Centrifugal and Centripetal autofrettage are discussed and compare.
\end{abstract}

Keywords: Thermal Autofrettage ; Re-Autofrettage; Spherical vessel ; Elastic perfectly plastic material ;FE Analysis; Residual stress;

\section{Introduction}

In recent decades, various methods have been proposed for strengthening the vessels. The autofrettage process is possibly the most well known method. Autofrettage is a process in which the component is subjected to a certain amount of pre-internal pressure so that its wall becomes partially plastic. The pressure is then released and the residual stresses lead to a decrease in the maximum von-Mises stress in the working loading stage. This means an increase in the pressure capacity of the component. Residual stresses are setup on unloading internally pressurized components subsequent to the application of high pressures such that the stress distribution beyond yield is observed in the component. A favourable distribution of residual stresses increases the strength of the pressure components. From[2-13] different researcher are given idea of autofrettage technique created by pressure, discussed re-autofrettage process, effect of bauschinger effect on residual stress for different material model and Fatigue life of autofrettage pressure vessel. Some of them done FE analysis of different autofrettage model.

Thermo elastic-plastic and residual stress analysis of Pressure vessel components are necessary for the nuclear, chemical, and military industries and also for fluid transmission and storage applications. In general, the existence of any temperature gradient across the wall of a thick-walled vessel induces a thermal stress. Often, thermal stresses are greater than those generated by application of either internal and or external pressure. From an economical point of view, the thermo elastic- plastic method is used for design of such vessels. Detailed analyses of thermal stress in spherical and cylindrical vessels in the elastic range are given in [14-17]. In [18] the behaviour of thick-walled spherical and cylindrical vessels under thermal and mechanical stresses is considered. The exact solution for the stress distribution in a thick-walled sphere made of elastic-perfectly plastic material and under a steady state, radial temperature gradient is obtained in [19]. In the same paper an approximate solution with negligible elastic strain is also examined; The onset of yield in thick-walled spherical vessels for various combinations of temperature and pressure and various radius ratios is studied in [20]. Elasticplastic thermal stresses in a spherical vessel under a temperature gradient across the wall thickness are studied in [21]. M.H. Kargarnovin A. Rezai Zarei, H. Darijani. discussed the Wall thickness optimization of thick-walled spherical vessel using thermo-elastic-plastic concept[22].

In particular, spherical pressure vessels, due to their inherent stress and strain distributions require thinner walls compared to cylindrical vessels; therefore, they are extensively used in gas-cooled nuclear reactors, gas or liquid containers rather than heads of close-ended cylindrical vessels. High thermal stress during loading can cause plastic deformation in the vessel and after the removal of temperature gradient(unloading) the residual stress is set. So, The autofrettage process can also be achieved by means of thermal stresses. In this analysis a Analytical solution for thermal autofrettage is derived. finite element analysis with ANSYS is performed on spherical vessel for thermal autofrettage and compared with mechanical autofrettage. 


\section{Theoretical Concept}

The maximum positive values of $\sigma_{t}$ occur in correspondence to the internal fiber if the flux is centripetal, and in correspondence to the external fiber if the flux is centrifugal. As the inner surface temperature increases for centrifugal flux the largest difference between $\sigma_{t}$ and $\sigma_{r}$ obviously occurs at the inner surface, just as for the internal pressurization problem. For centripetal flux also the largest difference between $\sigma_{t}$ and $\sigma_{r}$ occurs at the inner surface, but with the positive sign for the centripetal flux and the negative sign for the centrifugal flux. Thus, yielding similarly occurs first at the inner surface of the heated shell in common to both problems. Yield temperature increases as $\sigma_{y}$ increases and decreases as $\alpha, \mathrm{E}, \vartheta$ and $r_{o} / r_{i}$ increases.

For centrifugal flux as the inner surface temperature, Ti, increases keeping To fixed, first elastic stresses occur throughout the entire thickness then, yielding initiates at the inner surface. Next the yielding moves outward from the inner surface creating a plastic region between $r=r_{o}$ and $r=r_{c}$ (the elastic plastic interface radius),i.e., the region $r_{i} \leq r \leq r_{c}$. However, this plastic region does not just simply continue to grow until the entire shell is plastic as in the collapse analysis for inner- surface pressurization. The elastic- plastic interface radius is less than outside radius $\left(r_{c}<r_{o}\right)$. The yielding initiation behavior is identical to that for inner surface pressure. The plastic region expands outward with increasing temperature gradient until a second yield surface develops at the outer surface. The new plastic region begins to grow from the outer surface of the shell to inward. The two elastic- plastic boundaries grow closer as the temperature increases. For the centripetal flux also yielding initiates at the inner surface and then yielding moves outward from the inner surface creating a plastic region from $r=r_{o}$ to some growing radius $r=r_{c}$ in which $\left(r_{c}<r_{o}\right)$ until a second yield surface develops at the outer surface. So for centrifugal flux and centripetal flux yielding behavior is identical. The centrifugal residual hoop stress is positive at the inner surface and negative near the outer surface, its change sign from positive to negative between $r_{i}$ and $r_{o}$. The centrifugal residual radial stress is also positive sign and increase with temperature gradient. Moreover for mechanical loading $\sigma_{r}$ is always compressive and largest at the inner surface and throughout the rest of the shell (except $\sigma_{r}=0$ at $r=r_{o}$, the unpressurized surface ).But The circumferential hoop stress is always tensile throughout the shell and shifts from being largest at the outer surface to being largest at the inner surface as the pressure increases. $\sigma_{i}=\sigma_{t}-\sigma_{r}$ is always positive for internal pressurization. So residual stresses for centrifugal flux are not suitable for increase in the pressure capacity of the component even at high temperature gradient because this value is very less and for further mechanical loading it increase the circumferential hoop stress at the inner surface and component fail at inner surface earlier[Fig.1]. But for centripetal flux, residual stress can give the same benefit as mechanical autofrettage [Fig.5]. With centrifugal flux also residual stress can give positive result if the outside temperature is negative(lower than $-273^{\circ} \mathrm{C}$ ), but it is difficult to obtain below $-273^{\circ} \mathrm{C}$.

The strain -stress relations can be written in terms of the stresses, the coefficient of thermal expansion $(\alpha)$,the free-thermal strain and the plastic strain.

The differential equation of equilibrium for sphere, $\frac{\partial \sigma_{r}}{\partial r}-\frac{2}{r}\left(\sigma_{t}-\sigma_{r}\right)=0$.

The strain-compatibility equation,

$$
\begin{aligned}
& \frac{d \varepsilon_{t}}{d r}+\frac{\varepsilon_{t}-\varepsilon_{r}}{r}=0 \ldots \ldots \ldots \ldots \ldots . . .(2) \\
& \varepsilon_{r}=\frac{\partial u}{\partial r}=\frac{1}{E}\left(\sigma_{r}-2 v \sigma_{t}\right)+\alpha T \ldots \\
& \varepsilon_{t}=\frac{u}{r}=\frac{1}{E}\left[(1-v) \sigma_{t}-v \sigma_{r}\right]+\alpha T . . \\
& \frac{\partial}{\partial r}\left(2 \sigma_{t}+\sigma_{r}+\frac{2 E \alpha T}{(1-v)}\right)=0 \ldots \ldots \ldots . . .
\end{aligned}
$$

The steady state temperature in the case of spherical symmetry is given by the well-known formula

$$
T=T_{o}+\left(T_{i}-T_{o}\right) \frac{r_{o} / r-1}{r_{o} / r_{i}-1} \text {. }
$$

Accordingly to satisfy the Mises yield criterion'

$$
\sigma_{t}-\sigma_{r}=\sigma_{y} \ldots \ldots .(7)
$$


If two regions or zones( inner plastic zone and outer elastic zone) are created with increasing the outer surface temperature and keeping inside temperature fixed, then, The stresses for centripetal flux satisfying the yield criterion for inner plastic zone[24],

So, the radial stress determining differential equation become $\left(r_{i} \leq r \leq r_{c}\right)$.

$\int d \sigma_{r}=\int \frac{2 \sigma_{y}}{r} d r$

$\sigma_{r}=2 \sigma_{y} \ln \frac{r}{r_{i}}$

satisfying the boundary condition $\sigma_{r}=0$ at $r=r_{i}$, From equation (9) and yield criterion, $r_{i} \leq r \leq r_{c}$

$$
\sigma_{t}=\sigma_{y}\left(1+2 \ln \frac{r}{r_{i}}\right) .
$$

Now $\sigma_{r}$ and $\sigma_{t}$ is continuous at $r=r_{c}$

So, $\sigma_{r_{\text {elastic }}}=\sigma_{r_{\text {plastic }}}$ at $r=r_{c}$ and $\sigma_{t_{\text {elastic }}}=\sigma_{t_{\text {plastic }}}$ at $r=r_{c}$

to obtain

Elastic stresses in the elastic region $\left(r_{c} \leq r \leq r_{o}\right)$ are

$$
\begin{aligned}
& \sigma_{r}=-2 \sigma_{y} \frac{r_{c}^{3}\left\{1+r_{c} / r_{o}+\ln \left(r_{c} / r_{i}\right)\right\}}{r_{o}^{3}\left(2+r_{c} / r_{o}\right)\left(1-r_{c} / r_{o}\right)^{2}}\left(\frac{r_{o}^{3}}{r^{3}}-1\right)+2 \sigma_{y}\left(\frac{r_{c}}{r_{i}}-\frac{r_{c}}{r_{o}}\right) \frac{\left\{1-r_{c}^{3} / r_{o}^{3}+\ln \left(r_{c}^{3} / r_{i}^{3}\right)\right\}}{\left(2+r_{c} / r_{o}\right)\left(1-r_{c} / r_{o}\right)^{2}}\left(\frac{r_{o} / r-1}{r_{o} / r_{i}-1}\right) \cdots \cdots\left(r_{c}\right) \\
& \sigma_{t}=2 \sigma_{y} \frac{r_{c}^{3}\left\{1+r_{c} / r_{o}+\ln \left(r_{c} / r_{i}\right)\right\}}{r_{o}^{3}\left(2+r_{c} / r_{o}\right)\left(1-r_{c} / r_{o}\right)^{2}}\left(\frac{r_{o}^{3}}{2 r^{3}}+1\right)+2 \sigma_{y}\left(\frac{r_{c}}{r_{i}}-\frac{r_{c}}{r_{o}}\right)\left\{\frac{\left\{1-r_{c}^{3} / r_{o}^{3}+\ln \left(r_{c}^{3} / r_{i}^{3}\right)\right\}}{\left(2+r_{c} / r_{o}\right)\left(1-r_{c} / r_{o}\right)^{2}}\left(\frac{r_{o} / 2 r-1}{r_{o} / r_{i}-1}\right) \cdots\right.
\end{aligned}
$$

If three regions are created with centripetal flux,

Then within first plastic region $\left(r_{i} \leq r \leq r_{c}\right)$,

$$
\begin{aligned}
\sigma_{r} & =2 \sigma_{y} \ln \frac{r}{r_{i}} \ldots \ldots \ldots \ldots . . . . . \\
\sigma_{t} & =\sigma_{y}\left(1+2 \ln \frac{r}{r_{i}}\right) . . .
\end{aligned}
$$

After second yielding, an outer plastic region develops inward from the outer surface[Fig.2] .The radius to the boundary of the outer plastic region is $r_{f}$, where the stresses for centripetal flux satisfy the yield criterion

$$
\sigma_{t}-\sigma_{r}=-\sigma_{y} \ldots \ldots \ldots . . .(15) \quad r_{f} \leq r \leq r_{o}
$$

Again, the radial stress determining the equilibrium equation become.

$\int d \sigma_{r}=-\int \frac{2 \sigma_{y}}{r} d r$

$\sigma_{r}=-2 \sigma_{y} \ln r+\mathrm{c}$.

because $\sigma_{r}=0$ at $r=r_{o}$,

Thus the stresses in outside plastic zone are $\left(r_{f} \leq r \leq r_{o}\right)$

$$
\begin{aligned}
& \sigma_{r}=2 \sigma_{y} \ln \frac{r_{o}}{r} \ldots \ldots \ldots . . .(18) \\
& \sigma_{t}=\sigma_{y}\left(2 \ln \frac{r}{r}-1\right) \ldots \ldots \ldots . . .
\end{aligned}
$$

At The new elastic -plastic boundary $\left(r=r_{f}\right) \sigma_{r}$ must be continuous

$$
\left.\sigma_{r_{\text {elastic }}}\right|^{r=r_{f}}=\left.\sigma_{r_{\text {plastic }}}\right|^{r=r_{f}}
$$

The stresses in the middle elastic zone are

$$
\begin{aligned}
& \sigma_{r}=B\left[\frac{r_{o}^{3}}{r^{3}}-\frac{r_{o}^{3}}{r_{f}^{3}}\right]-\beta E\left(\frac{r_{o} / r-r_{o} / r_{f}}{r_{o} / r_{i}-1}\right)+2 \sigma_{y} \ln \frac{r_{o}}{r_{f}} \ldots . .(20) \\
& \sigma_{t}=-B\left[\frac{r_{o}^{3}}{2 r^{3}}+\frac{r_{o}^{3}}{r_{f}^{3}}\right]-\beta E\left(\frac{r_{o} / 2 r-r_{o} / r_{f}}{r_{o} / r_{i}-1}\right)+2 \sigma_{y} \ln \frac{r_{o}}{r_{f}} \ldots .(21)
\end{aligned}
$$


Where we do not know $\mathrm{B}$ and $\beta$ depends on $r_{f}$, which is function of $r_{c}$, but yielding occurs in the elastic region at both elastic -plastic boundaries ,

$$
\begin{array}{ll}
\sigma_{t}-\sigma_{r}=-\sigma_{y} & \text { at } r=r_{f} \\
\sigma_{t}-\sigma_{r}=\sigma_{y} & \text { at } r=r_{c}
\end{array}
$$

Finally, $\sigma_{r}$ must be continuous at the first elastic-plastic boundary, $r=r_{c}$.i.e.,

$$
\left.\sigma_{r_{\text {elassic }}}\right|^{r=r_{c}}=\left.\sigma_{r_{\text {plassic }}}\right|^{r=r_{c}}
$$

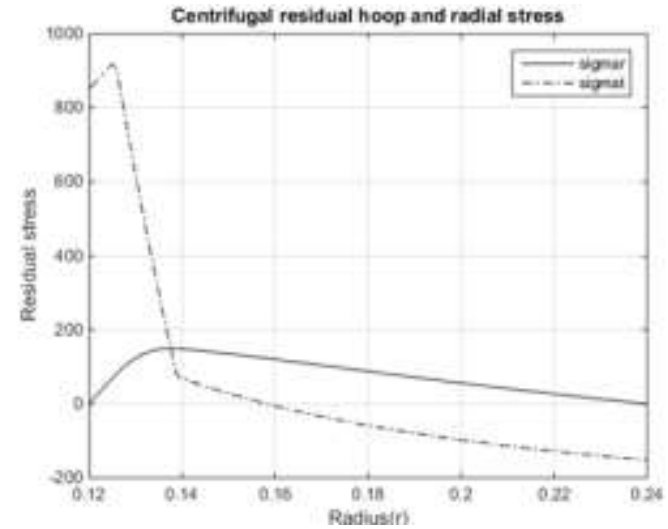

Figure .1: Centrifugal residual stresses at $\mathrm{T}_{\mathrm{i}}=572^{\circ} \mathrm{C}$ and $\mathrm{T}_{\mathrm{o}}=41^{\circ} \mathrm{C}$

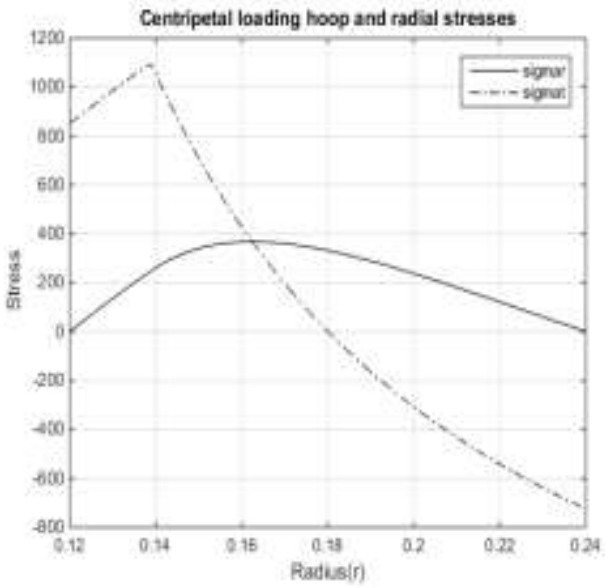

Figure.3: Centripetal loading stress at $\mathrm{T}_{\mathrm{i}}=41^{\circ} \mathrm{C}$ and $\mathrm{T}_{0}=572^{\circ} \mathrm{C}$

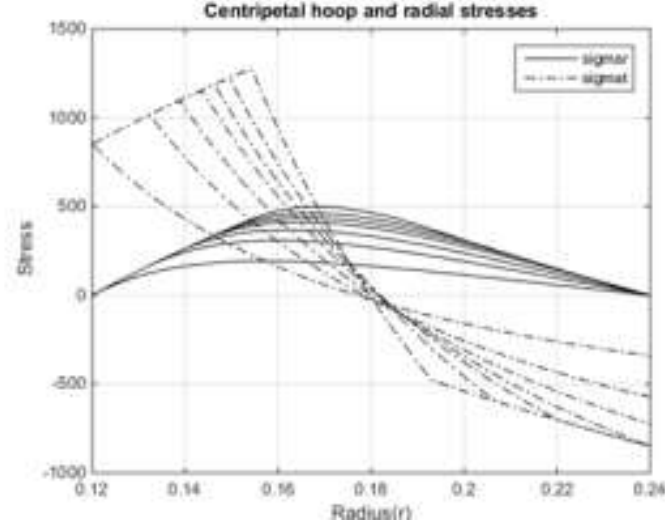

Figure .2: Centripetal hoop and radial stresses at various Temperature

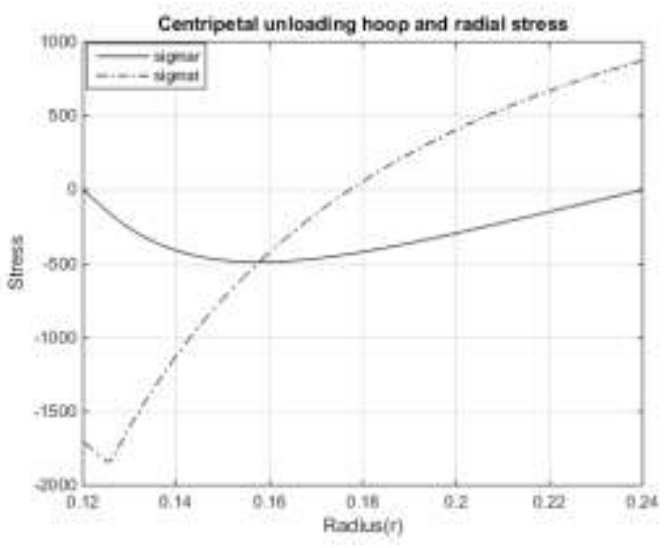

Figure.4: Centripetal unloading stress at $\mathrm{T}_{\mathrm{i}}=41^{\circ} \mathrm{C}$ and $\mathrm{T}_{0}=572^{\circ} \mathrm{C}$

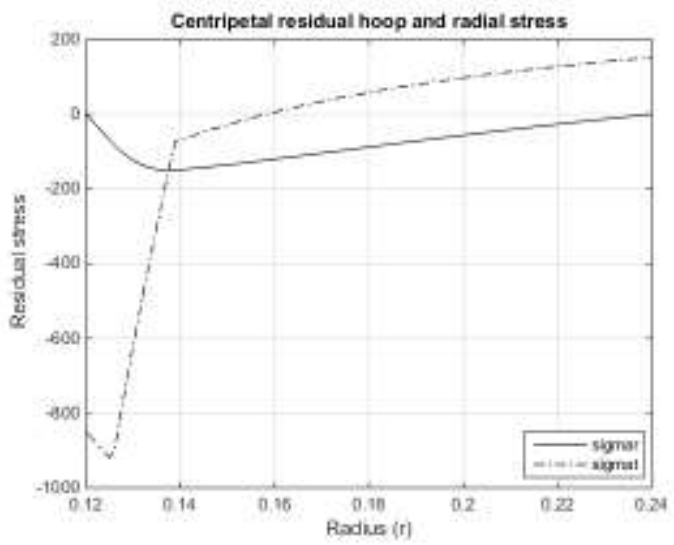

Figure.5: Centripetal residual stresses at $\mathrm{T}_{\mathrm{i}}=41^{\circ} \mathrm{C}$ and $\mathrm{T}_{\mathrm{o}}=572^{\circ} \mathrm{C}$ 
from which we get the following relation

$\ln \left(\frac{r_{c} r_{f}}{r_{o} r_{i}}\right)=\frac{2 r_{c}}{3 r_{f}}\left(\frac{r_{f}}{r_{c}}-1\right)^{2}$

So, finally stresses due to centripetal flux for middle elastic region are, $r_{c} \leq r \leq r_{f}$

$$
\begin{aligned}
& \sigma_{r}=-2 \sigma_{Y} \frac{2 r_{c}^{3} / 3 r_{o}^{3}}{\left(r_{c} / r_{f}\right)\left(1-r_{c} / r_{f}\right)}\left[\frac{r_{o}^{3}}{r^{3}}-\frac{r_{o}^{3}}{r_{f}^{3}}\right]+2 \sigma_{y}\left(\frac{r_{f}}{r_{i}}-\frac{r_{f}}{r_{o}}\right)\left(1+\frac{r_{c}^{2} / r_{f}^{2}}{\left(1-r_{c} / r_{f}\right)}\right)\left(\frac{r_{o} / r-r_{o} / r_{f}}{r_{o} / r_{i}-1}\right)+2 \sigma_{y} \ln \frac{r_{o}}{r_{f}} \ldots . .(23) \\
& \sigma_{t}=2 \sigma_{Y} \frac{2 r_{c}^{3} / 3 r_{o}^{3}}{\left(r_{c} / r_{f}\right)\left(1-r_{c} / r_{f}\right)}\left[\frac{r_{o}^{3}}{2 r^{3}}+\frac{r_{o}^{3}}{r_{f}^{3}}\right]+2 \sigma_{y}\left(\frac{r_{f}}{r_{i}}-\frac{r_{f}}{r_{o}}\right)\left(1+\frac{r_{c}^{2} / r_{f}^{2}}{\left(1-r_{c} / r_{f}\right)}\right)\left(\frac{r_{o} / 2 r-r_{o} / r_{f}}{r_{o} / r_{i}-1}\right)+2 \sigma_{y} \ln \frac{r_{o}}{r_{f}} \ldots . . .
\end{aligned}
$$

\section{Centripetal loading stress:}

Here maximum positive value of $\sigma_{t}$ in correspondence to the internal fiber and negative to the external fiber. Loading centripetal thermal hoop and radial stress is shown in Fig.3]. The radial stress is zero at the inner surface and at the outer surface. In between the inside and outside surfaces, $\sigma_{r}$ always tensile. Here if the outside temperature increases first yielding initiate at the inner surface and inner plastic region expand toward the outer surface. After a limit if again increase outside temperature secondary yielding is initiate at the outer surface and plastic region grows from the outer surface towards the inner plastic region. When two plastic regions meet such that the shell become fully plastic[Fig.2].

\section{Centripetal Unloading stress:}

For centripetal flux unloading radial stress is compressive and zero at inside and outside fiber. Here maximum negative values of $\sigma_{t}$ in correspondence to the internal fiber and positive to the external fiber [Fig.4].

\section{Centripetal residual stress:}

On cooling, a significant amount of compressive residual thermal hoop and radial stress are generated at and around the inner radius of the sphere. The resulting residual thermal hoop and radial stress is shown in Fig.5.At the outer elastic zone tensile residual hoop stresses of small value are generated. The compressive residual thermal radial stress are more compressive near the inner radius. The sphere do not undergo second stage of plastic deformation from outside radius due to the limitation on. $\left(T_{o}-T_{i}\right)$. Due to large amount of compressive stresses setup near the inner radius, the sphere is now able to withstand a high internal pressure than the normal without autofrettage sphere. The tensile hoop stress due to internal pressure loading get added to the compressive residual hoop stress generated near the inside bore and the resulting stress becomes smaller in magnitude. So, for centripetal flux residual stress can give the same benefit as mechanical autofrettage

\section{Comparison Of Analytical Results And The Finite Element Model}

An equivalent model was constructed in ANSYS, using the dimensions and a bilinear kinematic model taking $E_{t}=0$, which behave like Elastic perfectly plastic material. The material's constants are as follows: $E=206 \mathrm{GPa}$, Radius ratio, $\mathrm{k}=2, E_{t}=E_{t u}=0, \sigma_{y}=850 \mathrm{MPa}$, and $\vartheta=0.3$, Temperature gradient $\left(\mathrm{T}_{\mathrm{O}}-\mathrm{T}_{\mathrm{i}}\right)=531^{\circ} \mathrm{C}, \alpha$ $=17.1 \times 10^{-6}{ }^{\circ} \mathrm{C}$ The finite element $2-\mathrm{D}$ model of an autofrettaged sphere is constructed in ANSYS. A quadratic axis symmetric 8-node elements have been used for inelastic finite element analysis. The hoop and radial stress distribution through the thickness of the sphere has been evaluated in ANSYS for the same temperature gradient and then compared with the analytical model results. The centripetal thermal residual stress obtained from analytical elastic perfectly plastic model [Fig. 5.] are compared with the results of 2D FE model [Fig.6] with ANSYS. It is observed that The autofrettage stresses shows excellent agreement. There is negligible difference between the stresses obtain by analytical expressions and FE model. 


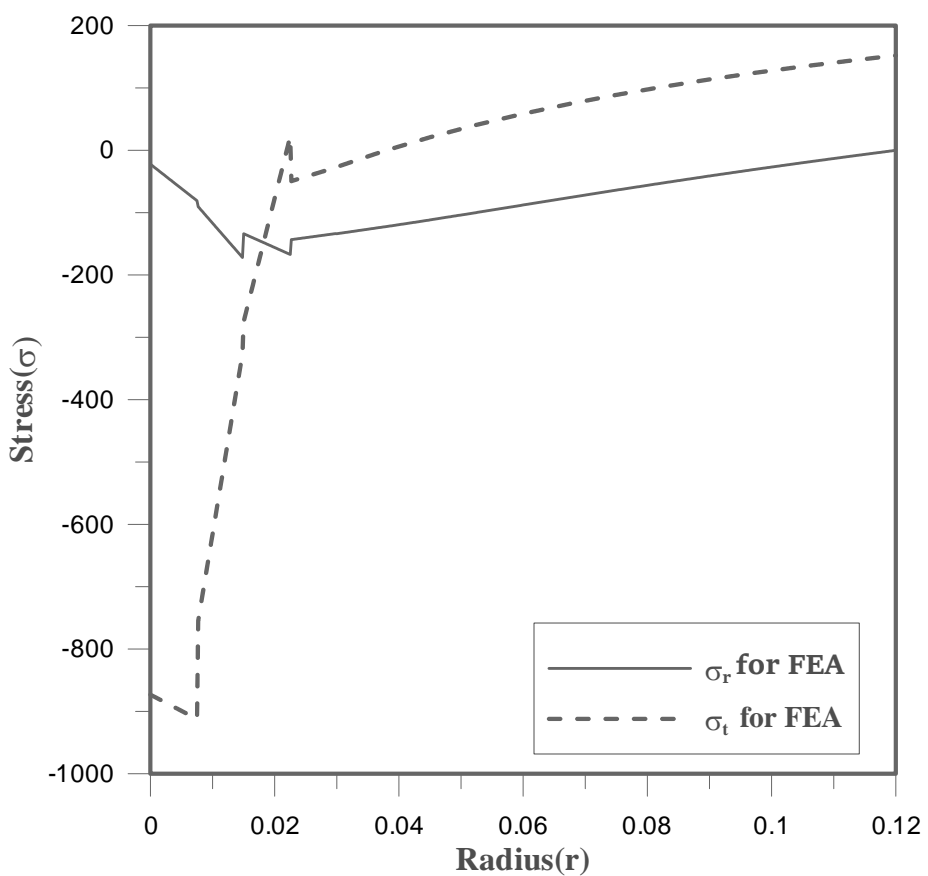

Fig.6:Residual centripetal thermal hoop and radial stress

\section{Conclusion}

1. Autofrettage procedure, previously proposed by mechanical load, may also be created by Thermal load .

2. Centripetal temperature gradient are more suitable for thermal Autofrettage.

3. If the temperature gradient exceeds some limit yielding at outside of the sphere, thus losing much of the potential benefit of autofrettage, because of the significant increase in tensile residual hoop stress at the outside diameter.

4. Elastic- plastic unloading increase residual stress near the bore than only elastic unloading.

5. Thermal autofrettage is a potential and simple process compare to conventional autofrettage created by mechanical load.

6. Thus, we must carefully determine the temperature at which the analysis is no longer valid because the material model and properties. That temperature depends on other physical properties of the specific material such as the melting temperature and how the various material properties degrade as the melting temperature is approached.

\section{Acknowledgements}

This work supported by Research Program supported by the Department of Mechanical Engineering, Jadavpur University, India.

\section{References}

[1]. Huang, X. P., and Cui, W., 2005, "Effect of Bauschinger Effect and Yield Criterion on Residual Stress Distribution of Autofrettaged Tube," Gun Tubes 2005 Conference, Oxford, April. 1.

[2]. Livieri, P., and Lazzarin, P., 2002, "Autofrettage Cylindrical Vessels and Bauschinger Effect: An Analytical Frame for Evaluating Residual Stress Distributions,” ASME J. Pressure Vessel Technol., 124, pp. 38-46.

[3]. R .Thumser,J.W.Bergmann, \& M.Vormwald, 2002, Residual stress fields \& fatigue analysis of autofrettaged parts ,International journal of Pressure Vessel and piping,79,113-117pp.

[4]. Parker, A. P., 2004, "A Re-Autofrettage Procedure for Mitigation of Bauschinger Effect in Thick Cylinders," ASME J. Pressure Vessel Technol., 126, pp. 451-454.

[5]. X. P Huang \& W. C. Cui , 2006 ,Effect of Bauschinger Effect and Yield Criterion on Residual Stress Distribution of Autofrettaged Tube," ASME J. Pressure Vessel Technol., Vol. 128, 212 -216.pp

[6]. Hamid Jahed, Babak Ahmadi Moghadam \&Mojtaba Shambooli, 2006, Re-Autofrettage," ASME journal of Pressure Vesse, Vol.128, 223-226pp.

[7]. C. Levy, M. Perl, S. Kotagiri, 2006, The Bauschinger effect's influence on the SIFs of multiple longitudinal coplanar cracks in autofrettaged pressurized cylinders , Engineering Fracture Mechanics(2006) (73),1814-1825pp.

[8]. A. P. Parker \& X. Huang, 2007, Autofrettage and Reautofrettage of a Spherical Pressure Vessel, Journal of Pressure Vessel Technology, Vol. 129, 83-88 pp.

[9]. R. Adibi-Asl1\& P. Livieri2,2007, Analytical Approach in Autofrettaged Spherical Pressure Vessels Considering the Bauschinger Effect, ," ASME J. Pressure Vessel Technol., 411-419 pp. Vol. 129.

[10]. M.H. Hojjati, A. Hassani, 2006, Theoretical and finite-element modeling of autofrettage process in strain-hardening thick-walled cylinders, International Journal of Pressure Vessels and Piping 84 (2007) 310-319pp. 
[11]. Milligan, R. V., Koo, W. H., and Davidson, T. E., 1966, “The Bauschinger Effect in a High Strength Steel,” J. Basic Eng., 88, pp. $480-488$.

[12]. Lee, H.-S., Yoon, J.-H., Park, J.-S., and Yi, Y.-M., 2005, “A Study on Failure Characteristic of Spherical Pressure Vessel,” J. Mater. Process. Technol., 164-165, pp. 882-888.

[13]. Gibson, Amer Hameed, Anthony P. Parker and John G. Hetherington, 2006, A Comparison of Methods for Predicting Residual Stresses in Strain-Hardening, Autofrettaged Thick Cylinders, Including the Bauschinger Effect ," Journal of Pressure Vessel Technology .,Vol. 128, 217-222pp.

[14]. Boley BA, Weiner JH. Theory of thermal stresses. New York: Wiley; 1960.

[15]. Nowaki W. Thermo-elasticity. Oxford: Pergamon Press; 1965.

[16]. Timoshenko S, Goodier JN. Theory of elasticity. New York: McGraw-Hill; 1951

[17]. Johnson W, Mellor PB. Engineering plasticity. London: Ellis Horwood; 1983.

[18]. Whalley E. The design of pressure vessels subjected to thermal stress-I, general theory for monoblock vessels. Can $\mathrm{J}$ Technol 1956;34(2):268-75.

[19]. Cowper JR. The eastoplastic thick-walled sphere subjected to a radial temperature gradient. Trans ASME 1960;27(3):496-500

[20]. Derrington MG, Johnson W. The onset of yield in a thick spherical shell subject to internal pressure and a uniform heat flow. Appl Sci Res Ser A 1958;7(4):408-14.

[21]. Johnson W, Mellor PB. Elastic-plastic behaviour of thick-walled spheres of non-work-hardening material subject to a steady state radial temperature gradient. Int J Mech Sci 1961;4(1):147-54.

[22]. M.H. Kargarnovin*, A. Rezai Zarei, H. Darijani. Wall thickness optimization of thick-walled sphericalvessel using thermo-elastoplastic concept. International Journal of Pressure Vessels and Piping 82 (2005) 379-385

[23]. Hamid Jaheda, Behrooz Farshib, Mohammad Hosseini,2006, Fatigue life prediction of autofrettage tubes using actual material behaviour, International Journal of Pressure Vessels and Piping $83,749-755$ pp.

[24]. J. Chakraborty, 2006, Theory of plasticity, Published by Elsevier Butterworth-Heinemann.

[25]. S.M. Kamal U.S.Dixit, (2015), Feasibility study of thermal Autofrettage of thick-walled cylinders, Journal of Pressure Vessel Technology137, 061207,1-17pp

\section{Nomenclature}

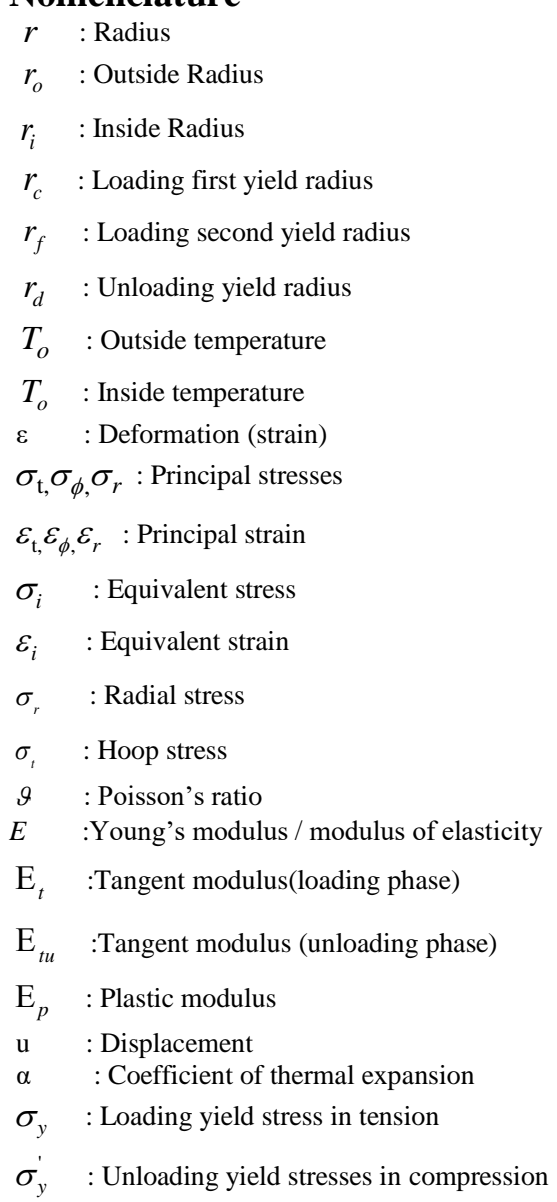

